\title{
Epidemiological Methods in Prevention of Blindness
}

\author{
D C MINASSIAN
}

London

\section{SUMMARY}

A major objective of the Prevention of Blindness programme of the World Health Organisation (WHO PBL Programme) is that by the year 1989 National Prevention of Blindness Programmes should be established in 60 developing countries which have a huge excess of avoidable blindness. To date, this has been achieved in 57 developing countries. Centres in a number of academic institutions have been designated "WHO Collaborating Centres for Prevention of Blindness". These are actively supported by the WHO PBL Programme to undertake collaborative survey and research work in developing countries. A principal aim of such work is to generate epidemiologically sound information that could form the bases for rational planning, implementation and proper evaluation of programmes for prevention of blindness.

This paper outlines the main epidemiological methods that have been employed recently, by a WHO collaborating centre based at the Institute of Ophthalmology in London, in population based eye surveys and in epidemiological research in a number of developing countries. The key aspects of the methodology are discussed in detail in the context of practical experience. The outcome of the studies are also mentioned briefly.

The three distinct types of epidemiological studies discussed are: (1) Population-based sample surveys, (2) Longitudinal cohort (follow-up) studies and (3) Hospital-based case-control studies.

According to the most recent estimates from the World Health Organisation, there are in the world between 27 and 35 million blind people who cannot count fingers held up at 3 meters i.e. visual acuity of less than $3 / 60$ in the best eye. Of the world's blind, $80 \%$ live in developing countries mostly in Asia (c. 20 million) and Africa (c.6 million) clustered largely in disadvantaged communities in rural areas and urban slums. The risk of blindness in many of these neglected communities is 10 to 40 times higher than that in industrially developed regions of Europe.

Prevalence data from blindness surveys show that about $50 \%$ of the blindness in many developing countries is primarily due to cataract, other major disease pathways being trachoma, onchocerciasis and blinding malnutrition. ${ }^{1}$ Thus, up to $80 \%$ of the blindness in developing areas is potentially avoidable, that is it could be cured or prevented within the limits of resources which could be reasonably made available.

In recognising the huge overburden of avoidable blindness in developing countries, the World Health Organisation's Prevention of Blindness Programme is actively supporting collaborative work, including epidemiological and operational research, to assess the magnitude and distribution of blindness and blinding disease and the need for ophthalmic services, and to identify and measure the relative importance of major risk factors that might be amenable to preventive control measures. The aim of such collaborative work is to generate the necessary information so that national and regional programmes for prevention of blindness could be planned, implemented and evaluated in a rational manner with due regard to the actual needs of the communities and to epidemiologically determined priorities.

To these ends, a number of Centres in various academic institutions have been designated as 
"WHO Collaborating Centres for Prevention of Blindness". Some of these Centres have been particularly active in collaborative work involving surveys and epidemiological research in developing countries. This paper reviews the epidemiological methods used mainly in the context of recent work conducted by ICEH, a WHO Collaborating Centre and a Department of the Institute of Ophthalmology in London.

\section{National Survey of Blindness and Eye Disease in the Gambia}

The Gambia, a small country on the west coast of Africa, provides a useful model for many parts of Africa as it has one ophthalmologist in charge of an Eye Unit serving the total population of c.800,000. A population based prevalence survey of blindness and eye disease was conducted in March to May 1986 on a country-wide sample. ${ }^{2}$ The survey was initiated by the Ministry of Health in the Gambia, in collaboration with the International Centre for Eye Health, London and the Prevention of Blindness Programme of the World Health Organisation.

The aims of the survey were to provide the necessary base-line data on causes and distribution of blindness and serious eye disease throughout the country to assist in the development of a National Eye Care Programme, and, subsequently to evaluate its impact on the prevalence of blindness.

\section{Designing the Survey:}

The sampling procedure and a detailed protocol of work were designed by a working group as a practical exercise in epidemiology and planning during the 1986 Course in Community Eye Health at ICEH. The planning group comprised several ophthalmologists from various developing countries including the senior ophthalmologist from the Gambia who was to take overall charge of the survey, a number of ophthalmic trained nurses and assistants some of whom were to take part in the field work, and, members of staff including a health economist with experience in Africa, an ophthalmologist who had extensive expertise in management of ophthalmic services and training in Africa, and an ophthalmic epidemiologist (the author of this article).
Designing the survey required detailed largescale maps, recent census data listing all the villages and larger communities with their population size, and, most importantly, expert local knowledge of the demography. The composition of the planning group enabled good access to all of these.

Having defined the objectives of the survey in terms of the various estimates of blindness and its causes that were required, the first question that arose concerned the sample size. Since the number of people who should be examined would partly depend upon the methods used in selecting the sample, the first task was to design a sampling method that would tend to minimise sampling errors for a given sample size, thus minimising the number of people that should be examined. To achieve this, distinct sub-groups of the population were defined so that within each sub-group (strata) the prevalence of blindness from the major causes was expected to be fairly homogeneous across the village communities (settlements), and so that the differences in prevalence were expected to be maximal between the strata. For example, settlements of a particular size North of the river in the Eastern Medical Administrative region were regarded as a separate stratum distinct from settlements of similar size South of the river in the same region, mainly because of differences in ease of access to the ophthalmic services in the capital city of Banjul. Similarly, the urban population of Banjul was considered as a separate stratum distinct from the rural communities. Another factor used for "stratification" of the population was the size of the rural settlements. Having defined the various strata, a fairly small sample could then be taken from each stratum. The sample sizes from each stratum had to be proportional to the population size of the stratum in order to avoid over-representation of a particular stratum in the final sample.

Ideally, for optimal precision, an appropriate number of individual persons would be selected at random from the total population of each stratum. Clearly this was impracticable because of the difficulties in (a) preparing a complete list of all individuals with their addresses so that a number could be selected randomly, and (b) the extensive travel arrangements and costs in locating and examining the selected individuals. The usual compromise was made to randomly 
select from each of the strata, clusters of people who lived in one or more closely linked communities.

All members of each selected cluster would then be examined and would constitute the final sample. This could be achieved fairly easily since recent census data provided a complete list of all settlements together with their population size for the whole country, enabling construction of a sampling frame i.e. a complete list of all the clusters from which a certain number of clusters would be randomly selected.

Travelling costs in terms of money and time demanded further compromises of this kind. The selection of the sample would be carried out in three main stages starting with selection of two administrative districts from each of the three Medical Administrative Regions that covered the whole country. Thus the clusters in the first stage of the samplings were the districts. In the second stage, settlements would be selected randomly from each of the chosen districts. The third stage involved further sampling of compounds (extended households) from the larger settlements. All the residents of the selected compounds would then be included in the sample and examined.

These compromises meant that a larger sample size would be required to maintain the level of precision desired in estimating the prevalence of blindness etc. In general, the larger the clusters, the bigger the sample required. However, the multistage cluster random sampling design would make it relatively easy to locate and examine the extra numbers.

There were three other remaining main factors upon which the sample size would depend: (a) The desired precision of the estimates of blindness and its causes, (b) the probability that the desired precision is achieved, and (c) the actual prevalence of blindness in the population. The latter would be the minimum prevalence expected and was defined as $0.5 \%$. The desired precision was set in terms of a maximum sampling error of $\pm 0.2 \%$ and the probability of achieving the precision was chosen to be $95 \%$. These specifications together with the relevant details of the sampling design were then entered into a computer programme (developed at ICEH) which provided a list of the sample sizes that would be required from each stratum. The computations were based on equations in WG
Cochrans "Sampling Techniques".3 The total sample size required was computed to be at least 8,000 persons.

Selection and identification of the villages through multistage cluster random sampling was achieved by using the computer programme which was fed with the necessary census and demographic information. The sampling procedure in Banjul involved selection of clusters of people who lived in distinct street blocks.

Having produced a list of all the settlements that had to be visited, the travelling and other arrangements were planned and budgeted in detail.

The composition and number of survey teams and of the advance enumeration team would depend, importantly, upon the nature and volume of information that had to be recorded for each person in the sample. It was decided to use the standard WHO Eye Examination Record (version II) which was developed primarily for eye surveys in developing countries. (The author was a member of the WHO workshop in which the eye examination record was formulated).

\section{The Field Work and Outcome of the Survey:}

The survey and enumeration teams were trained on location during a four week period prior to the survey. In particular, the ophthalmologists and ophthalmic assistants responsible for clinical examinations and tests, were trained in standard methods of examination and recording of information. This included a pilot survey during which the degree of agreement between the examiners in grading inflammatory trachoma was evaluated.

Regular meetings throughout the survey helped to maintain standards and quality of work. These were organised and supervised by Dr Hanna Faal, the senior ophthalmologist in charge of the survey, who was also the field manager.

Much of the success of the survey depended upon the enumeration team who were usually the front line of contact with the communities. The four members of the team were experienced in census work and were of local origin with intimate knowledge of the local culture. Other factors that contributed to the success of the survey included the provision of on the spot treatment including eye medication and referral 
of more serious cases for surgical or medical treatment to the base hospital.

In the event, the sample provided 8,696 persons of whom 8,174 were examined, giving a good overall coverage of $94 \%$. As expected, the worst coverage occurred in Banjul (86\%).

The findings of the survey together with recommendations for priority oriented action have been reported to the Ministry of Health in Banjul and to the WHO. ${ }^{4}$ The survey data were able to estimate the number of persons in urgent need of cataract surgery and lid surgery (for trachomatous entropion) in various regions of the country. The number with low vision requiring spectacles was also estimated by region. The data indicate that about half of the blindness in the Gambia, mainly in the rural populations, is potentially remediable through the provision of cataract surgery and appropriate spectacles.

There is now in the Gambia an active programme of training and expansion of the ophthalmic services in the base hospital and into the rural areas. These have been planned within the limits of the resources that can be made available in the country with some additional help and ongoing commitment from the Royal Commonwealth Society for the Blind.

A major objective of the Prevention of Blindness programme of the World Health Organisation is that national prevention of blindness programmes should be established in 60 developing countries by 1989 . So far this has been achieved in 57 of them.

\section{Other Surveys:}

Other population-based surveys of blindness and eye disease based on methods similar to those used in the Gambia survey have been conducted in the Lama Kara District of Togo in West Africa (1987) and in Thailand. 5,6 The first nation-wide surveys in Thailand sampled the rural population in 1982 and was repeated in 1987 to measure the changes in prevalence, distribution and patterns of blindness. Both surveys were conducted by Thai ophthalmologists trained at ICEH. The survey data have been helpful in formulating the National Prevention of Blindness Programme in Thailand.

A regional survey designed at ICEH has been planned for the Chaiyaphum Province in North East Region of Thailand to provide more detailed information that could be used in planning of a regional programme. The survey should also provide the necessary base-line for subsequent evaluation of the curative and preventive work in the Province.

\section{Epidemiological Studies of Incidence and Risk Factors in Cataract}

Cataract is the commonest blinding condition and constitutes the main surgical workload in ophthalmic services throughout the world. There are an estimated 17 million people blind from cataract in the world. ${ }^{7}$ This major health problem is believed to be much more prevalent in many developing countries where it causes visual disability at an earlier age. The magnitude of the overburden of cataract in the developing world is indicated by comparison of the findings from a survey in the Punjab in India with the findings from the population study in Framingham USA. $^{8}$ The age-standardised prevalence of cataract in persons aged 52 and older was almost three times higher in the Punjab.

The challenge in most developing countries is to evolve ophthalmic services that can deliver safe cataract surgery and aphakic correction to the urban as well as the neglected rural populations with a high rate of uptake by the visually disabled. The rational planning of such services requires not only prevalence data that estimate the backlog of cataract but also incidence data that estimate the number of new cases that occur in a given time period, e.g. per annum. Furthermore, epidemiological research methods could be used to identify the factors that contribute to poor (and good) uptake of the ophthalmic services, to evaluate the costeffectiveness of alternative strategies of intervention and, to assess the impact of the services on the socio-economic status and on eye health of the communities.

A second but equally important challenge is development of practicable preventive measures. Thus, high priority is given to epidemiological research to identify and measure the relative importance of major adverse risk factors (and possibly protective factors) that influence the risk of cataract blindness, so that preventive strategies can be devised.

There are three main types of epidemiological methods that could be used for these purposes: 
(1) Longitudinal Cohort (follow-up) Studies:

In longitudinal studies an appropriate random sample of the adult population in a defined area would be examined and interviewed to generate base-line data including clinical data and information on exposure to the risk factors under study. The sample would then be followed up for say five to 10 years and systematically reassessed so that the age specific incidence of cataract and visual loss in a given period of time could be estimated for the population as a whole, and for various sub-groups of the population that are more or less exposed to the risk factors (or protective factors). The word "exposed" is used in a wide sense so that it could mean being a female of a particular socio-economic status, or it could equally well refer to those who are chronically malnourished or have suffered a particular illness or condition that is suspected to enhance (or possibly diminish) the risk of cataract. The incidence (or risk) of cataract could then be compared between the sub-groups (cohorts) who are similar in most respects, including age and sex, except in respect of exposure to a particular factor. If the risk of cataract is found to be significantly higher in the "exposed" group compared to their not-exposed or less exposed controls, then, with due caution, the excess risk could be attributed to the exposure, provided that known or suspected sources of possible bias have been adequately taken into account.

Nothing much can be done to ensure even distribution of unknown or unsuspected factors between the comparison sub-groups in the sample, thus there always remains some chance that the real culprit is a factor other than the blamed one. This is in contrast to the situation in properly randomised large-scale clinical trials where alternative treatments (including placebo) are allocated randomly to a large number of patients. The randomisation process in a large sample tends to result in even distribution of all prognostic factors, known and unknown, between the various treatment groups.

The assessment and control of confounding in longitudinal studies as in other types of epidemiological research is of fundamental importance and could usually be achieved through careful attention to detail in design, conduct and, very importantly, proper statistical analysis of the study to ensure that comparisons of risk are made between groups that are as far as possible similar in all relevant respects except for the particular exposure factor under study.

The two important comparative measures of risk that can be estimated from longitudinal studies (and from case-control studies in certain situations) are Relative Risk and Population Attributable Risk. Relative risk is simply the ratio of two risks (or two incidences when these are proportions). Usually the risk of the particular disease or disability in the exposed group is divided by the risk in the not-exposed or less exposed group. Thus, a relative risk of one indicates no association between the exposure to the risk factor and the risk of the disease since the incidence of the disease is the same in both groups irrespective of exposure. Relative risk of two would indicate that the exposed are two times more likely to develop the disease since the incidence of the disease in the exposed is twice that in the other group. Relative risks of less than unity would indicate that the exposure was in fact protective rather than damaging.

The relative risk is thus a good measure of the strength of the association between the exposure factor and the disease or disability. Population attributable risk (or ethiologic fraction) is the proportion of the disease in the population that can be attributed to (caused by) the exposure factor. This is a useful estimate of the magnitude of the disease or disability that might be prevented if the risk factor was eliminated. It can be misleading unless the association between risk factor and disease is a causal one.

Clearly longitudinal studies are impracticable for rare diseases since vast numbers would have to be followed up for long periods in order to harvest adequate numbers of disease cases. Other disadvantages include high cost and more likelihood of loss of both subjects and investigators during the long period of study. Furthermore, it is difficult to maintain the same standard procedures for examination and interview over a long period. However, visually disabling cataract in an appropriately aged population (say 40 to 60 years old) is not a rare phenomenon in many locations in developing countries.

During the past six years, a so far successful longitudinal investigation has been in progress in Madhya Pradesh in Central India. The study is 
the first of its kind in the developing world. Its main objectives are

(a) To provide a directly measured estimate of age-specific incidence of cataract in males and females,

(b) To provide comparative estimates of mortality in relation to cataract,

(c) To identify and evaluate the relative importance of a number of risk factors, including dehydrational crises and malnutrition, that may substantially enhance the risk of blinding cataract in that population.

A further objective was to obtain certain population based estimates that could be used to enhance the validity of a rapid concurrent casecontrol investigation based in the eye hospital.

One of the important objectives of the longitudinal and the concurrent case-control studies was to measure for the first time, the extent by which the risk of blinding cataract is enhanced by exposure to dehydrational crises from severe life-threatening cholera-like diarrhoeal disease and from heatstroke. Convincing biochemical evidence from John Harding's work at the Nuffield Laboratory of Ophthalmology in Oxford indicated that such dehydrational crises may play a part in cataractogenesis through cyanate-induced carbamylation of lens proteins. ${ }^{9}$ Harding postulates that other possibly damaging effects of severe diarrhoeal disease may operate through osmotic stress, acidosis and malnutrition. ${ }^{10}$ However, whereas such laboratory data pointed to a possible "cause" of cataract, they could not answer the question: "By how many times is the risk of blinding cataract higher in the exposed compared to those not exposed to the dehydrational crises?", and, perhaps more importantly, the question: "What proportion of the blinding cataracts in the population is caused by the dehydrational crises?". The epidemiological studies employed were designed to answer both questions in terms of Relative Risk and Population Attributable Risk respectively.

The longitudinal study was designed at ICEH and is conducted in collaboration with the ophthalmologist in charge of the Chattisgarh Eye Hospitals in Raipur and his team. The first phase of the study (1981-82) during which the baseline data were accumulated was supported by the RCSB. The subsequent and current phases are supported through the WHO Prevention of Blindness Programme.

\section{Outline of Method:}

(i) Examinations to identify incident cases

In Raipur District, 11 villages were selected randomly from the Mandir-Hasud Sector of Arang Block which has a population of c. 100,000 . The total population aged 30 and older in each village was examined and interviewed during the survey in 1981-82. Clinical examinations included assessment of visual acuity and refractive error, ascertainment of the principal cause of visual loss if any and, grading of central lens opacities using a rapid method that has been shown to have good inter-observer agreement. ${ }^{11}$ The clinical examinations were conducted by two ophthalmologists. In addition, two ophthalmic trained paramedics with special training in grading of cataract participated in the grading of central lens opacities. The cataract grading method would have been inadequate had we wanted to detect small changes in the area or density of opacification in the lens. For such purposes objective documentation of the lens with photographic and image analysis equipment would be required. ${ }^{12,13,14,15,16}$ The field method used was practicable and adequate for our purposes. We wished to detect clinically important deterioration of the image forming properties of the lens whereby little or no central lens opacity in persons with good vision progress to substantial central lens opacities that reduce vision to less than $6 / 18$ (or worse). This was because we wanted to measure the risk of visually disabling or blinding cataract in the various sub-groups. We and most people in the study villages were less interested in trivial lens changes that would not be expected to have an appreciable adverse effect on vision.

The same standard procedures were used to examine the sample five years later in 1986-87, to identify the incident cases of visually disabling or blinding cataract.

\section{(ii) To ascertain exposure status}

Experience of severe life-threatening diarrhoeal disease in the memorable past was as important to ascertain as that which might occur in the five year follow up period, since carbamylation damage to lens proteins are believed to be cumulative. The questions on past experience of such severe illness were formulated and tested in the rural and urban populations over a period of six months prior to the studies. More than 600 
persons of various ages and educational and cultural backgrounds were interviewed during the pilot study. The definitive questions focused on severe life-threatening cholera-like diarrhoeal disease, severe enough to render the patient seriously ill in bed for at least three days, an event that should have been memorable. Both the remembered frequency of separate episodes of such severe disease and the occurrence (in relation to age or some memorable calendar event) were recorded.

The contents of the questions and a flowdiagram to show the standard sequence are reported elsewhere. ${ }^{17}$

No doubt, in some instances the response would be unreliable and would not reflect the true level of exposure. However, this would tend to mask or minimise the estimate of excess risk in the exposed and would not result in overestimates of relative risk, provided that every individual was subjected to the same standard interview procedure with no undue prodding or short-cuts. Great care was taken to minimise this potential serious source of bias. All the interviews were conducted by a health worker who had been trained specifically for the task and extensively tested in the standardised method.

Other topics in the interview included questions about history of heatstroke which was a well recognised condition in the community, main sources of water supply, dietary habits and socio-economic indicators such as income, occupation and caste.

The data on occupation together with locations of main residences and duration of residence in each location, measurements of altitude, and the type of terrain local to the sample were used to estimate the exposure to ultraviolet radiation in sunlight as this might possibly have been a risk factor for cataract and therefore a potential confounder. We had no reliable data on sunlighthours or average daily erythemal units for the locality. However, apart from the occupation, there was very little variation in the sample.

Weight and height were measured so that an index of nutritional status could be arrived at. Whereas these were not always true indicators of nutritional status in every individual, a subgroup comprising persons with weight/height far below average of the sample would have a much higher proportion of individuals with back- ground of malnutrition compared to a sub-group in which all individuals had weight/height close to or above average. This would allow stratification of the sample by two or three levels of exposure to malnutrition.

\section{Outcome of the Longitudinal study:}

In the first phase of the study, 1,742 persons aged 30 or older were examined and interviewed. More than $80 \%$ of these were successfully relocated five years later and reassessed. These included 110 persons who had died. In almost all of the 345 mostly young persons who could not be relocated, we obtained reasonably reliable evidence that they were alive and that they had not become visually disabled or blind. This was done through interviews with family members and friends. The main reason for being absent was travel for permanent or temporary work.

The data are at present being analysed through both classic methods of stratified analysis to control confounding and by more recently developed methods of multivariate modelling.

This type of study which involves detailed interviews in a survey situation could not have been implemented adequately without the goodwill and co-operation of the village communities. The team from Chattisgarh Eye Hospital lead by Dr Vijay Mehra were well known and respected by the communities. As in the Gambia survey, the team were equipped to provide minor treatments for eye and other complaints and to refer those who required hospital based care.

\section{(2) Case-Control Studies:}

In contrast to longitudinal studies, case-control investigations are highly suited to study of aetiology in rare diseases and are often the only practicable option. Ideally, the study is conducted in a hospital or clinic that has a well established catchment population so that the cases (persons with the particular disease or disability) who attend may reasonably be assumed to represent a sample of the incident cases that occur in the catchment population during the study period. The exposure experiences of the cases are ascertained and compared to that in the controls (non-cases who attend during the same period).

Many factors may influence the probability of attendance in cases and in controls so that the 
sample may be far from a randomly selected one from the total incident cases in the catchment population. However, this would not be expected to cause bias if the probability of attendance was influenced in the same way and to the same extent in both cases and controls.

Usually, the incidence of the disease or disability in various sub-groups cannot be measured in a case control study. However, the odds ratio may be computed and regarded as a good estimate of relative risk in appropriately designed studies. The population attributable risk may also be computed when a fairly reliable estimate of the proportion of exposed persons in the population is available. Thus, the two most important comparative measures of risk can sometimes be estimated with reasonable reliability in appropriately designed case-control studies.

The obvious advantages of case-control studies are that they can be completed in a relatively short period of time (usually 1-3 years) with relatively low cost, particularly if they are based in hospitals that have a large turnover of the cases under study. However, they can be extremely difficult to design and invariably require great care and expertise in formulating a protocol that is free of ambiguity, particularly in the criteria for diagnosing a case, and in the procedure for selection of appropriate controls.

Probably, the commonest serious methodological error that I have come across in reviews of some recent case-control studies is the inappropriate exclusion of controls from the study. In every such situation reviewed, the study findings were seriously invalidated because of the error.

There are many other potential sources of serious bias, including the phenomenon of confounding, that may result in gross overestimate (or underestimate) of relative risk in case-control studies. Some of the more important of these are discussed in the practical context of our collaborative work concerning risk factors in cataract.

The first of a series of case-control studies in the States of Madhya Pradesh and Orissa was conducted in the Chattisgarh Eye Hospital in Raipur. The hospital provides, free of charge, a high quality eye-care service to a well established catchment population which comprise a mix of urban and rural communities.
The primary objective of the study was to compare the unbiased and unconfounded risk of cataract in persons exposed to dehydrational crises from severe cholera-like diarrhoeal disease or heatstroke, with those not exposed (or less exposed) to such crises.

In a 12-month period all adult patients aged up to 59 who attended the hospital were categorised as cases (cataract) or possible controls according to clearly defined criteria based upon grade of central lens opacities and the accompanying visual impairment. Each case was individually matched with one control, who was selected as the next incoming non-case that satisfied the matching rules. Close matching for factors that might increase the risk of cataract and also be associated with dehydrational crises was of prime importance in minimising bias due to confounding.

The matching criteria were age, sex, occupation, residence and social caste. The pairmatching method of selecting controls was employed in order to minimise possible confounding in a relatively small sample. The alternative would have been to carry out the matching in the analysis stage whereby the data would be grouped into strata so that within each stratum cases and controls would have the same or similar age, sex etc. Relative risks could then be computed for each stratum and a weighted average relative risk calculated for the whole data. This approach would require a much larger sample size. However, great effort and time are required to ensure unbiased and proper selection of the control member of each matched pair in studies such as the Raipur case-control study. The alternative method is often easier to implement in spite of the much larger sample size. Furthermore, if pair-matching is done at the time of selection of controls, then the factors matched for cannot be evaluated as risk factors. Pair-matching was not employed in the subsequent case-control studies.

The upper age limit of the sample was set at 59 because of the rapid deterioration in the quality of response to the questions on remembered episodes of severe life-threatening diarrhoeal disease in persons aged 60 and over. This became apparent in the pilot survey and remained a consistent problem throughout the population-based longitudinal study.

As in the longitudinal study, clinical examina- 
tions including grading of cataract were conducted by two ophthalmologists and the interviews were carried out by a health worker trained specifically for this task. The interviews took place in a specially prepared separate room where it was easier to maintain the same standard procedure and environment throughout the study. This helped to ensure that the sensitivity and specificity of the diarrhoea questions in arriving at the truth were similar in both cases and in controls, so that even if the interview was a relatively poor diagnostic procedure, this would not constitute a serious source of bias. Inconsistencies in the interview technique could easily constitute the biggest source of serious bias in the study.

Similar bias could arise if the cataract cases had stronger motives to respond positively to the interview questions. For example, if the cataract cases were led to believe that the diarrhoea question was asked because it was suspected to have caused their eye problem, they would be expected to make more effort to remember past experience of such disease and therefore be more likely to recall it. Furthermore, they may be more inclined to exaggerate the severity of any past episodes of diarrhoeal disease. In order to guard against such "Recall bias", the diarrhoeal questions were hidden amongst many other topics of interview conveying the impression that a general health profile was being compiled for the patient.

Ideally, the interviewer should have been completely masked so that he would not know whether he was interviewing a case or a control. This would be desirable even when the honesty of the interviewer is beyond reasonable doubt. It was not possible to mask the interviewer in every case because some of the patients had severe visual disability with rather obvious cataracts that could have been recognised by the highly observant and intelligent interviewer. Nevertheless, there was no evidence of bias from this source. ${ }^{18}$ Masking of the examiners was achieved by conducting the clinical assessments prior to the interviews.

The pool of potential controls was not restricted to any one particular diagnostic category. They consisted mainly of patients with refractive errors, viral and other conjunctivitis and lachrymal problems. It was fundamentally important not to exclude any of the potential con- trols who for some or other apparent reason might have been expected to have positive history of dehydrational crises. To do so would almost certainly have led to serious overestimation of the importance of dehydrational crises as a risk factor in cataract.

\section{Outcome of the Raipur Case-Control Study:}

Three hundred and twenty patients with a mean age of about 44 were matched in pairs to produce 160 case/control pairs. Statistical analysis appropriate to matched-pair data show a strong and "dose-dependent" association between cataract and remembered episodes of dehydrational crises from severe cholera-like diarrhoeal disease or heatstroke. An approximate estimate of the population attributable risk indicated that nearly $40 \%$ of the cataract in the catchment population aged up to 59 might be attributable to dehydrational crises. Details of the findings are reported elsewhere. ${ }^{18}$

\section{Other Case-Control Studies:}

A second case-control investigation was conducted in an eye clinic within a general practice in the town of Titlagarh in Orissa State in India. The study included more than 1,600 patients who attended the practice during a period of 24 months. Pair-matching in selection of controls was not used. Instead, stratified data analysis and multivariate modelling techniques were employed to control confounding and detect effect modification. There were substantial differences in the environmental and sociocultural characteristics of the population in this study compared to the Raipur population. In spite of this and of the differences in methodology, the findings in the two studies were very similar. Thus, the population attributable risk was estimated to be $38 \%$, very close to the estimated $40 \%$ in Raipur. ${ }^{17}$ A similar but expectedly less strong association between cataract and severe diarrhoeal disease has been found in a case-control study in Oxford. ${ }^{19}$

A third collaborative case-control investigation, funded by the WHO Prevention of Blindness Programme, is at present in progress in Madhya Pradesh. The objectives of this study include assessment of genetic markers that could be risk factors, confounders or effect modifiers. If they are found to be so, then it would be important to include them in evaluation of other 
risk factors in future studies of cataract aetiology.

Recently we have used a case-control method in Somalia to investigate alterations of the anterior lens capsule in relation to exposure to ultraviolet irradiation from sunlight, as measured by the grade of climatic keratopathy. The study was conducted in an eye unit of the district hospital in Afgooye which is a small town near the capital city of Mogadishu. The eye unit was established as a joint project of the Government of Somalia and the non-governmental agency the Christoffel Blindenmission, in partnership with the International Centre for Eye Health of the Institute of Ophthalmology in London. The capsule changes investigated were confined to the central pupillary area and consisted of white opalescence ("frosting"), plateau formation and herniation of the lens capsule through the pupil ("bagging"). The latter two changes interfered severely with vision and were strongly associated with climatic keratopathy. ${ }^{20}$

The Raipur case-control study was funded by the Royal Commonwealth Society for the Blind, UK, the Orissa study was funded by the British Council for Prevention of Blindness who also supported the study in Somalia.

\section{References}

'World Health Organisation Programme for the Prevention of Blindness. Available data on blindness (update 1987). WHO/PBL/87.14.

${ }^{2}$ Faal H, Minassian D, Sowa S, Foster A. National survey of blindness and low vision in the Gambia - Results. Br J Ophthalmol (in press).

${ }^{3}$ Cochran WG. Sampling techniques. 3rd ed. New York. John Wiley \& Sons, 1977: 428.

${ }^{4}$ Faal H, Minassian D. National survey of blindness and eye disease in the Gambia: results and recommendations. Report to the Ministry of Health, the Gambia. 1986.

${ }^{5}$ Epidemiologic study of blindness and eye disease in Thailand 1983. Report to Ministry of Health, Thailand.

${ }^{6}$ Nandavisai C. Survey of blindness and it's major causes in Thailand 1987. Report to Ministry of Health, Thailand.

${ }^{7}$ Dawson CR, Schwab IR. Epidemiology of cataract: a major cause of preventable blindness. Bull WHO 1981; 59: 493-501.
${ }^{8}$ Chatterjee A, Milton RC, Thyle S. Prevalence and aetiology of cataract in Punjab. Br J Ophthalmol 1982; 66: 35-42.

${ }^{9}$ Harding JJ, Rixon KC. Carbamylation of lens proteins: a possible factor in cataractogenesis in some tropical countries. Exp Eye Res 1980; 31: 567-71.

${ }^{10}$ Harding JJ. Possible causes of the unfolding of proteins in cataract and a new hypothesis to explain the high prevalence of cataract in some countries. In: Regnault F, Hockwin O, Courtois $\mathrm{Y}$, eds. Aging of the lens. Elsevier/North Holland. 1980: 71-80.

"Mehra V, Minassian DC. A rapid method of grading cataract in epidemiological studies and eye surveys. Br J Ophthalmol (in press).

${ }^{12}$ Maclean H, Taylor CJ. An objective staging for cortical cataract in vivo aided by patternanalysing computer. Exp Eye Res 1981; 33: 597-602.

${ }^{13}$ Hockwin O, Dragomirescu V, Laser H. Measurement of lens transparency or its disturbances by densitometric image analysis of Scheimpflug photographs. Graefe's Arch Clin Exp Ophthalmol 1982; 219: 255-62.

${ }^{14}$ Chylack LT, Lee MR, Tung WH, Cheng HM. Classification of human senile cataractous change by the American co-operative cataract research group method: 1. Instrumentation and technique. Invest Ophthalmol 1983; 24: 424-31.

${ }^{15}$ Chylack LT, Cheng HM, White O. Retroillumination and Topcon SL-45 photography of cataracts in vivo: a quantitative study of the variances of these techniques with computerised image analysis. Invest Ophthalmol 1984; 25: 270. ARVO Abst Suppl.

${ }^{16}$ Sparrow JM, Bron AJ, Brown NAP, Ayliffe W, Hill AR. The Oxford clinical cataract classification and grading system. International Ophthalmol 1986; 9: 207-25.

${ }^{17}$ Minassian DC, Mehra V, Verrey J-D. Dehydrational crises: a major risk factor in blinding cataract. $\mathrm{Br} J$ Ophthalmol (in press).

${ }^{18}$ Minassian DC, Mehra V, Jones BR. Dehydrational crises from severe diarrhoea or heatstroke and risk of cataract. Lancet 1984; i: 751-3.

${ }^{19}$ van Heyningen R, Harding JJ. Do aspirin like analgesics protect against cataract? A casecontrol study. Lancet 1986; i: 1111-13.

${ }^{20}$ Johnson G, Minassian D, Franken S. Alterations of the anterior lens capsule associated with climatic keratopathy. $\mathrm{Br} J$ Ophthalmol (in press). 